\title{
Control-informed geometric optimisation of wave energy converters
}

\author{
Jean-Christophe Gilloteaux ${ }^{\star}$ John Ringwood
}

\author{
National University of Ireland, Maynooth, Co. Kildare, Ireland.
}

\begin{abstract}
This paper concerns the interplay between the physical geometry of a wave energy converter (WEC) and the control strategy adopted for the converter, with the ultimate aim of optimising the energy output of the device. An energy-based performance function is employed and we attempt to perform numerical optimisation of a heaving buoy employing a latching control strategy. We allow both draught and radius of the axisymmetric buoy to be adjusted using a numerical optimisation. A linear time-domain hydrodynamic program is used in order to simulate the device motion, while the optimization problem is solved by means of a simplex method. Results show the difference in the frequency response of an optimal buoy for a particular sea-state designed with and without knowledge of the control system.
\end{abstract}

Keywords: Wave energy; Point absorber; Device optimization; Latching control; Random sea.

\section{INTRODUCTION}

Traditionally, WECs are designed independently of the control system (McCabe et al., 2009; Alves et al., 2007; Babarit and Clement, 2006b), with the focus being on (control-free) optimisation of the geometry of the device for the prevailing sea conditions. In essence, this can involve matching the frequency response of the device to the average spectral description of the wave environment. It is well known that control techniques, such as latching, have the ability to change the characteristics of a WEC, allowing it to achieve a resonance other than natural. However, since latching only has the ability to slow down the dynamics of the WEC, the resonant frequency of the WEC must be placed well above the predominant sea condition if energy is to be optimally captured over the range of sea states encountered over a typical year. However, exactly where the resonance frequency of the WEC should be placed is a complex function of the statistical sea state description, the device hydrodynamics and the exact nature of the latching strategy employed.

The present work is part of a project aiming at the economical optimization of a real wave energy device. This paper presents preliminary results with a simple geometry in order to validate the optimisation program and to get straightforward results as part of the learning process of this kind of problem. This can be seen as an illustrative case study where we investigated the influence of latching control on the geometric optimization of a generic heaving device under the action of random waves. That is why some simplifications were used such as the device dynamics which is described by one-degree of freedom (heave motion), or the fluid-structure interaction solved by means of a linear potential program while the numerical optimization is performed with a simplex algorithm.

The paper layout is as follow: we first introduce the gov-

\footnotetext{
^ Corresponding author. E-mail: jc.gilloteaux@eeng.nuim.ie
}

erning equations of the problem, then the optimization procedure is described in details. Eventually, results are presented in order to show effect of considering control on a sample WEC and clearly demonstrate the difference in the frequency response of an optimal buoy for a particular wave climate designed with and without knowledge of the control system.

\section{MATHEMATICAL MODELLING}

\subsection{Body dynamics}

A generic point absorber is considered for the purpose of this study. The body is a generic point absorber represented as a truncated vertical cylinder with a diameter D and a draught $d$. We have allowed the device to oscillate vertically under the action of excitation forces, radiation damping forces and restoring forces as depicted on Fig. 1. The fluid is considered homogeneous, uncompressible, inviscid and with an irrotational flow.

According to Cummins (1962), the dynamics of such a system is governed by the following integro-differential equation

$$
\begin{aligned}
& \left(M+\mu_{\infty}\right) \ddot{\zeta}(t)+\int_{0}^{t} \dot{\zeta}(\tau) K(t-\tau) d \tau+B_{P T O} \dot{\zeta}(t)+K_{H} \zeta(t) \\
& =F_{e x}(t)
\end{aligned}
$$

where

- $\mu_{\infty}$ is the added mass at infinite frequency

- $M$ the mass of the cylinder

- $K$ the impulse response for the radiation forces

- $K_{H}$ the hydrostatic stiffness

- $B_{P T O}$ the power take-off damping coefficient

- $F_{e x}$ the excitation forces 


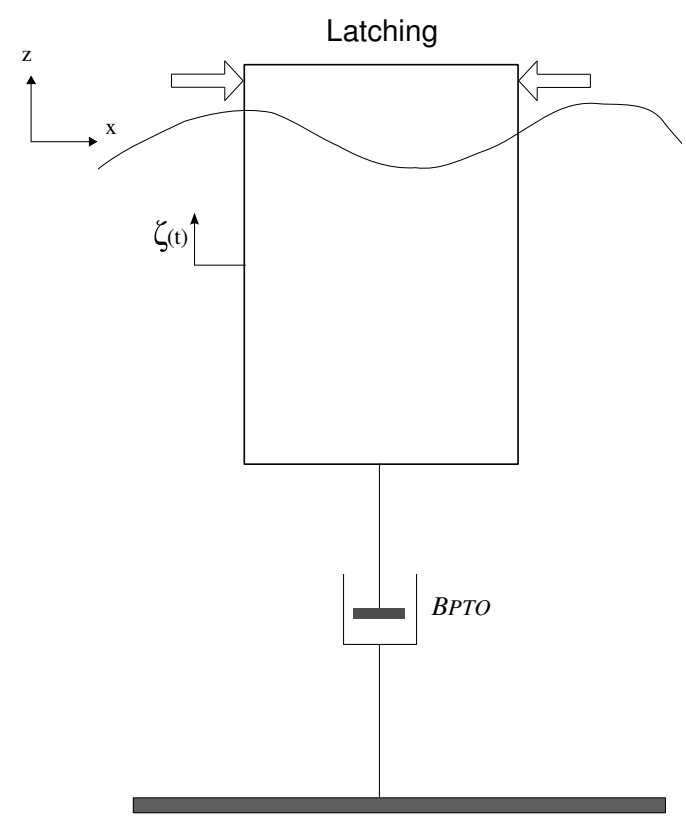

Fig. 1. Schematic of the generic heaving buoy.

The calculation of the hydrodynamical coefficients $\mu_{\infty}$, $K$, and $K_{H}$ were performed by using the time-domain seakeeping code ACHIL3D (Clément, 1997).

\subsection{Absorbed power}

Since PTO modelling is beyond the scope of the study, the power take-off was modelled as a linear damping force $F_{P T O}$ (cf. Fig. 1) as follow

$$
F_{P T O}=-B_{P T O} \dot{\zeta}
$$

while this is not ideal for WEC analysis, this approximation is usually used in wave energy and has already been used several times in the past (Babarit et al., 2004; Babarit and Clement, 2006a; Alves et al., 2007; Ricci et al., 2006) when the focus is on other issues. Consequently, the mean power $\hat{P}$ absorbed by the device over a time $\mathrm{T}$ is

$$
\hat{P}=\frac{1}{T} \int_{0}^{T} B_{P T O} \dot{\zeta}(\tau)^{2} d \tau .
$$

\subsection{The resource}

The excitation force in Eq. 1 was derived by discretizing the ITTC spectrum model (Rawson and Tupper, 1992) as follows

$$
S(\omega)=\frac{A}{\omega^{5}} \exp \left(\frac{-B}{\omega^{4}}\right)
$$

with

$$
\begin{gathered}
A=173 \frac{H_{S}^{2}}{T_{1}^{4}} . \\
B=\frac{691}{T_{1}^{4}} .
\end{gathered}
$$

and parameterised by the significant wave height $H_{S}$ and the mean period $T_{1}$.
The free surface elevation $\eta$ is modeled as a linear superposition of monochromatic waves, by sampling the energy spectrum $S(\omega)$ by a constant frequency step. One can synthesize a representative sample of the waves for a sea state defined by the couple $\left(H_{S}, T_{1}\right)$ by using

$$
\eta(t)=\sum_{j=1}^{N \omega} a\left(\omega_{j}\right) \cos \left(\omega_{j} t+\phi_{j}\right) .
$$

where $a\left(\omega_{j}\right)$ corresponds to the wave amplitude of the $j^{\text {th }}$ frequency component given by

$$
a\left(\omega_{j}\right)=2 \sqrt{S\left(\omega_{j}\right) \delta \omega}
$$

where $\omega_{j}$ and $\phi_{j}$ are circular frequency and phase of the $j^{\text {th }}$ wave component respectively, and where phase are randomly determined.

According to Cummins (1962), the corresponding excitation force introduced in Eq. 1 is then given by

$$
F_{e x}(t)=\int_{0}^{t} K_{e x}(t-\tau) \eta(\tau) d \tau
$$

where $K_{e x}$ is the impulse response in excitation computed with ACHIL3D.

\section{OPTIMIZATION PROCEDURE}

A Nelder-Mead simplex algorithm (Nelder and Mead, 1965) was used for resolving the optimization problem. This derivative-free deterministic algorithm has been chosen for its flexibility and robustness for solving complex optimization problems. The all optimization procedure is described on Fig. 2. First, the simplex algorithm evaluates the initial shape predefined by the user. Then, the seakeeping program is used for computing the hydrodynamic parameters used in Eq. 1. This program is considered as a black box, it first generates a mesh of the shape and then solves the hydrodynamic problem in the time-domain. Once all the hydrodynamic parameters are known, the next stage is the simulation of device motion under the action of random waves defined by the power spectrum $S(\omega)$ as defined in Eq. 4. The simulation stage finished, the objective function is then evaluated as the average power absorbed by the device during the simulation (Eq. 3 ). Eventually, the objective function is evaluated by the simplex routine which calculates a new radius and draugth for the next iteration if and only if the convergence criterion is not fulfilled.

This optimization procedure has to be performed automatically, that is why criteria have to be implemented in the program for tuning the different input parameters to the device dimension. In addition to deal with different shape, these criteria have to be optimal insofar as they have to provide both a simulation time as short as possible and results as accurate as possible.

Hence, different criteria were used during the different stages of the optimization procedure. Regarding ACHIL3D, the position of the center of gravity $Z_{G}$ has to fulfill 


$$
Z_{G}<Z_{B}
$$

where $Z_{B}$ corresponds to the center of buoyancy, in order to get the hydrostatic stability. One has to notice that this criterion does not affect the optimization as only heave motion is considered here.

Regarding the hydrodynamic parameters, ACHIL3D has to solve the hydrodynamic problem as fast as possible while guaranteeing a very good accuray. Accuracy is laid down the refinement of the mesh that discretizes the body and down the simulation duration over which the hydrodynamic problem is solved. Since both mesh refinement and simulation duration depend on body dimension, they have to be automatically computed by the optimization program. Concerning the simulation duration, ACHIL3D uses a non-dimensional simulation duration $T_{\text {sim }}^{*}$ defined as follow

$$
T_{\text {sim }}^{*}=T_{\text {sim }} \sqrt{\frac{g}{d r a u g h t}}
$$

where $T_{\text {sim }}$ is the dimensional simulation duration defined by the user and $g$ the gravitational constant. So, we decided to evaluate a non-dimensional simulation time for a vertical cylinder with regards to its dimensions. We have then run several simulations with different geometries until a simulation duration that satisfy both requirements in terms of accuracy and simulation time was found.

Regarding the mesh refinement, a similar approach was used. We have run several simulations with different mesh refinements and for one particular geometry until a convergence was reached. We have then considered the corresponding mesh refinement as a reference $n_{\text {mesh }}^{\text {ref }}$ from which all the mesh refinements $n_{\text {mesh }}$ will be deducted as follow

$$
n_{m e s h}=n_{m e s h}^{r e f} \frac{S_{w}}{S_{w}^{r e f}} \times 100
$$

where $S_{w}$ corresponds to the wetted surface of the body for which $n_{m e s h}$ is being calculated and $S_{w}^{r e f}$ to the wetted surface of the body of reference.

For the simulation part, a criterion was also used to tune the damping value $B_{P T O}$ of the power take-off to device dimensions. Hence, the following relation was used

$$
B_{P T O}=\frac{5}{100} \times M
$$

Regarding the optimization problem itself, the objective of the Nelder-Mead algorithm was to maximize the mean absorbed power $\hat{P}$ ( $c f$. Eq. 3), or in more suitable form for an optimization problem, the objective was to minimize the energy function $\hat{P}^{*}$ defined as follow

$$
\hat{P}^{*}=-\frac{1}{T} \int_{0}^{T} B_{P T O} \dot{\zeta}(\tau)^{2} d \tau
$$

Hence, the present optimization problem is a monoobjective optimization problem with two parameters: radius and draugth which have to be strictly positive.

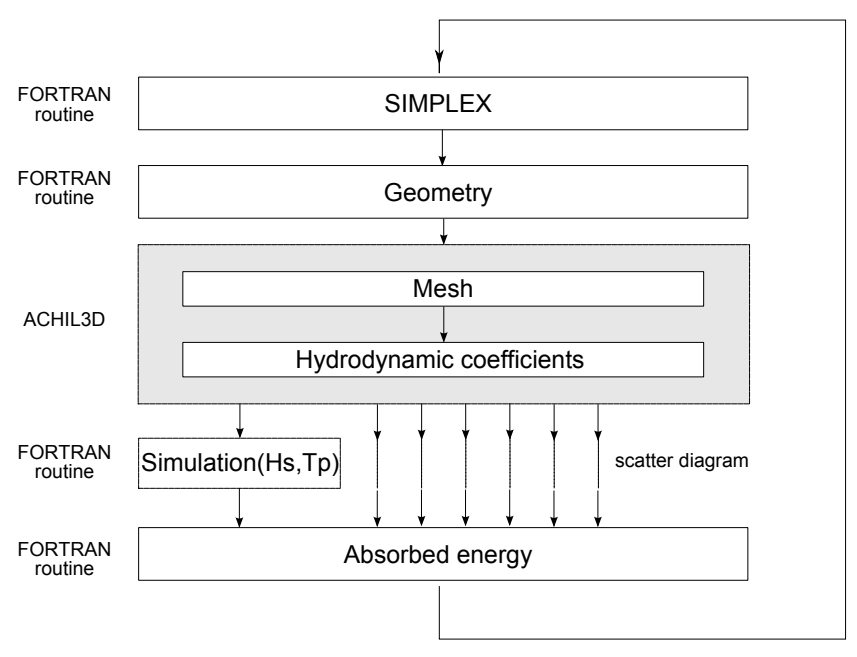

Fig. 2. Flow diagram of the optimization program

\section{RESULTS}

The results presented in this section come from optimizations carried out by using an Intel quad-core $/ 2.4 \mathrm{GHz}$ processor giving a computational time about a week for each of them. The non-dimensional simulation duration used to evaluate the hydrodynamic coefficients was $T_{\text {sim }}^{*}=13 \mathrm{~s}$, device motion was simulated over $600 \mathrm{~s}$ while the average absorbed power $\hat{P}$ was calculated over the last $300 \mathrm{~s}$ in order to get rid of the transient motion. The sea-state used here and defined by Eq. 4 had a significant wave height $H_{S}=3.5 \mathrm{~m}$, a mean period $T_{1}=9.14 \mathrm{~s}$ and 150 components $\left(N_{\omega}\right)$ were used for sampling the sea spectrum. Phases were pre-computed once in order to be exactly the same for every simulation of both optimization problems.

\subsection{Optimization with a passive PTO}

On figures 4, 5, 6, and 7, optimizations results with a passive PTO are presented where the evolution of average power absorbed, natural resonance period, radius and draught are plotted as a function of iteration, respectively. On figure 5, one can see that the optimization algorithm converges to a device with a natural resonance period close to the mean period of the sea-state. This result was expected since mean period (also called energy period $T_{E}$ ) is defined as the period of the spectrum where the energy is maximal in average. One can also notice that simplex method seems to be well suited for this kind of optimization problem as it converges relatively fastly despite initial conditions far to the optimal ones.

\subsection{Optimization with latching control}

In this section, optimization results with latching control are presented. We chose latching control because it is the simplest way of creating parametric resonance within the dynamics of the device in order to improve its efficiency (Budal et al., 1982). Under the action of a passive PTO, the device catches energy only from the wave components whose frequency lays in the bandwidth of the system. The principle of latching control principle is to lock the motion of the body when its velocity vanishes at the end of one oscillation, and waiting (over a time that has to be 
determined) for the most favorable situation to release the body.

While in regular waves optimal latching control can be determined semi-analytically (Babarit, 2005), under the action of random waves it is not as trivial (Eidsmoen, 1998) and it is necessary to define an appropriate strategy to determine when the body must be latched (Babarit and Clement, 2006a). Here, we chose to use both the instantaneous zero-crossing period $T_{z}^{i}$ and the natural resonance period $T_{r}$ to determine the latching time $T_{L}$ over which the cylinder will be locked, as follow

$$
T_{L}=\frac{T_{z}^{i}-T_{r}}{2}
$$

where the natural resonance period of the vertical cylinder $T_{r}$ is defined as

$$
T_{r}=\sqrt{\frac{K_{H}}{\left(M+\mu_{\infty}\right)}}
$$

and where $T_{z}^{i}$ is the instantaneous zero-crossing period corresponding to the period of the next zero-upcross wave by assuming that we are able to forecast the free surface elevation over $10 \mathrm{~s}$.

On figures 8, 9, 10, and 11 optimization results with laching control are presented, where the evolution of average power absorbed, natural resonance period, radius and draught are plotted as a function of iteration, respectively.

Table 1. Optimization results

\begin{tabular}{c|ccc} 
& $\operatorname{Tr}(\mathrm{s})$ & Radius $(\mathrm{m})$ & Draft $(\mathrm{m})$ \\
\hline No control & 9.14 & 12.52 & 14.08 \\
Latching & 6.22 & 6.38 & 6.19 \\
\hline
\end{tabular}

Table 1 summarizes the optimization results for both optimization problems. It shows that both optimal geometries are completly different and are characterized by two different natural resonance periods. Fig. 3, compares transfer functions also called Response Amplitude Operators (RAO) of the optimal devices between each other and with the power spectrum. It shows that, for this particular case i.e. with a heaving vertical cylinder, a linear PTO, a specific sea-state, and with latching control, the device geometry is highly control dependent. It highlights the fact that numerical optimization with control may play an important role in the sense that it may change the original natural resonance period of the device. In the optimization without latching, the optimal device obtained is not optimal if one want, afterwards, to apply latching control on it. The same remark can be stated for the optimization with latching, the natural mechanic properties of the optimal shape are not longer optimal for the specific sea-state, nevertheless with latching control, one can be sure that performance will be optimal. Similar results were already outlined by Babarit (2005), who optimized by means of a genetic algorithm the shape of the SEAREV wave energy device with and without latching control and where the optimization program ends up with two slighty different geometries.

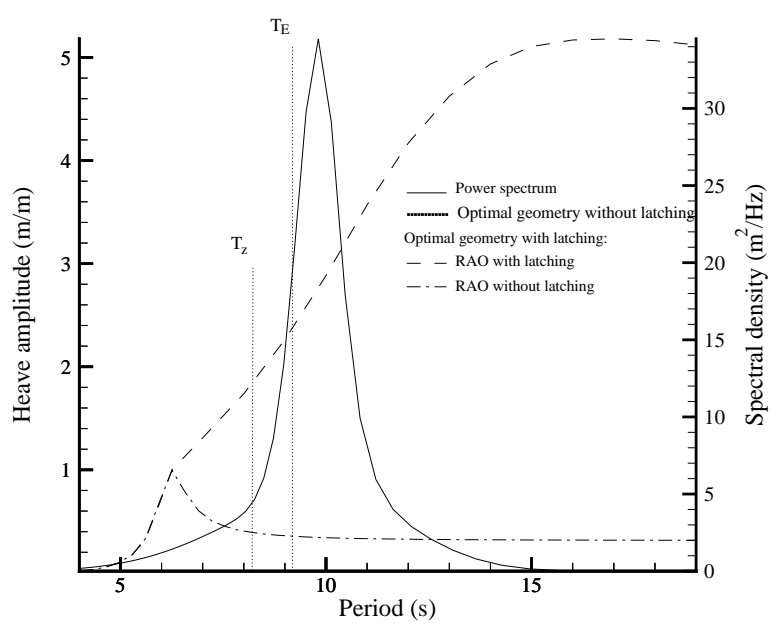

Fig. 3. Plot of power spectrum and RAOs for both optimal geometries obtained with and without latching control.

\section{CONCLUSION}

In the present paper a procedure was presented for the geometric optimization of a generic heaving wave energy device under the action of random waves. The optimization procedure was splitted into several stages, and used a linear potential program for computing the hydrodynamic coefficients. The optimization problem was solved by means of a simplex algorithm and used an energy based cost function.

Results for two optimization problems were presented. First, the shape of a vertical cylinder was optimized for a particular sea-state while a passive PTO was considered. Then, a second optimization problem was presented where latching control was used instead of passive loading. Results show that the optimal shape is relatively control dependent and both optimization problem end up with two geometries with different mechanical properties. It points out that, with regards to mechanical principle, seastate properties, PTO and control strategy, the control dependence of wave energy devices should be checked when shape optimization is performed. Finally, in the present study only latching control was used, other control strategies should arguably give different results, nevertheless likely effects are not trivial and differ regarding the type of WEC and PTO, that is why when one want to perform a geometric optimization of a real device all those parameters must be take into consideration.

\section{ACKNOWLEDGEMENTS}

The authors are grateful for the financial support provided by Enterprise Ireland.

\section{REFERENCES}

Alves, M., Traylor, H., and Sarmento, A. (2007). Hydrodynamic optimization of a wave energy converter using a heave motion buoy. EWTEC (Porto).

Babarit, A. (2005). Optimisation Hydrodynamique et Contrôle Optimal D'un Récupérateur de L'énergie Des Vagues. Ph.D. thesis, Ecole Centrale de Nantes. 


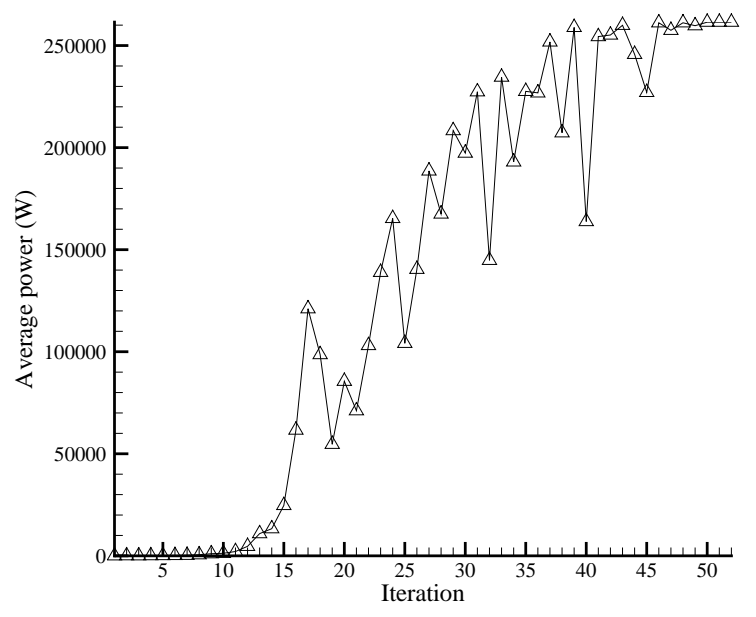

Fig. 4. Evolution of the average power with a passive PTO.

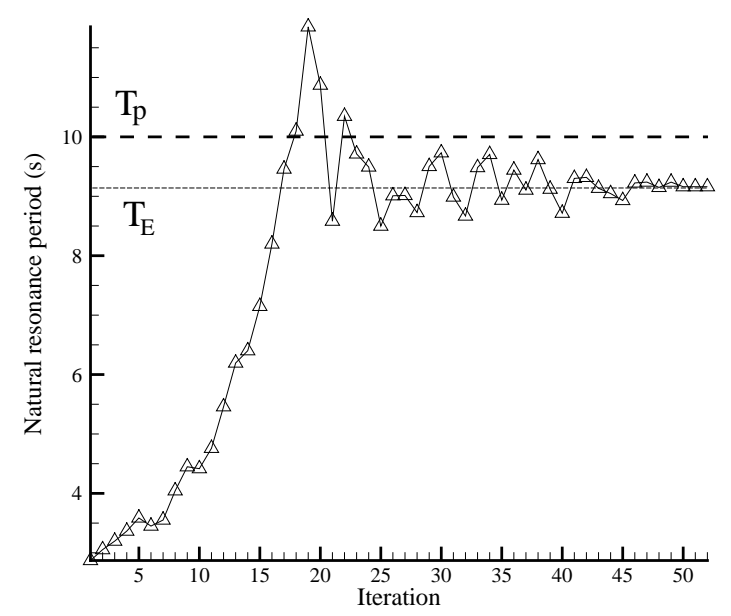

Fig. 5. Evolution of the resonance period with a passive PTO.

Babarit, A. and Clement, A. (2006a). Optimal latching control of a wave energy device in regular and irregular waves. Applied Ocean Research, 28, 77-91.

Babarit, A. and Clement, A. (2006b). Shape optimisation of the searev wave energy converter. In World Renewable Energy Congress IX.

Babarit, A., Duclos, G., and Clement, A. (2004). Latching control of power take off oscillator carried by a wave activated body.

Budal, K., Falnes, J., Iversen, P., Lillebeken, G., Oltedal, T., Hals, T., Onshus, T., and Hoy, A. (1982). The norwegian wavepower buoy project. The Second International Symposium on Wave Energy Utilization, 323-344.

Clément, A. (1997). Hydrodynamique instationnaire linéarisée : 'mise en oeuvre d'une méthode de singularités utilisant un modèle différentiel de la fonction de green.'. Technical Report LHN-9703, Laboratoire de Mécanique des Fluide de l'Ecole Centrale de Nantes.

Cummins, W. (1962). The impulse response function and ship motions.technical report 1961. Schiffstechnik, 9,

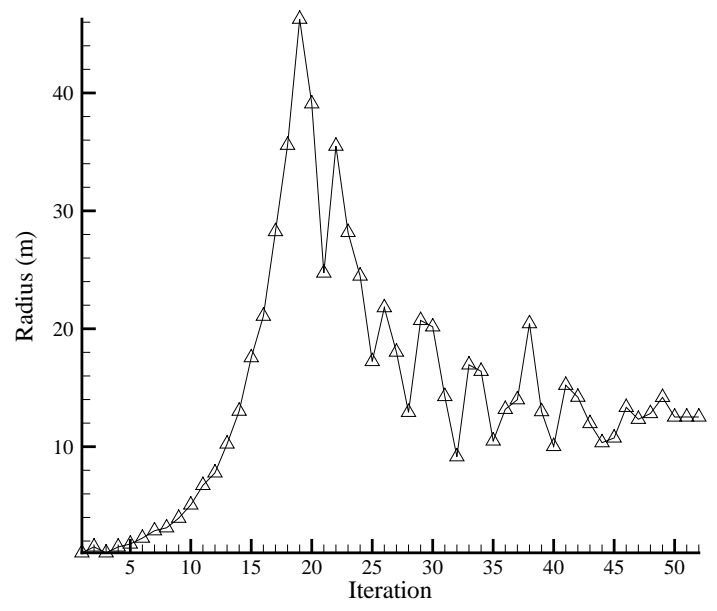

Fig. 6. Evolution of the radius with a passive PTO.

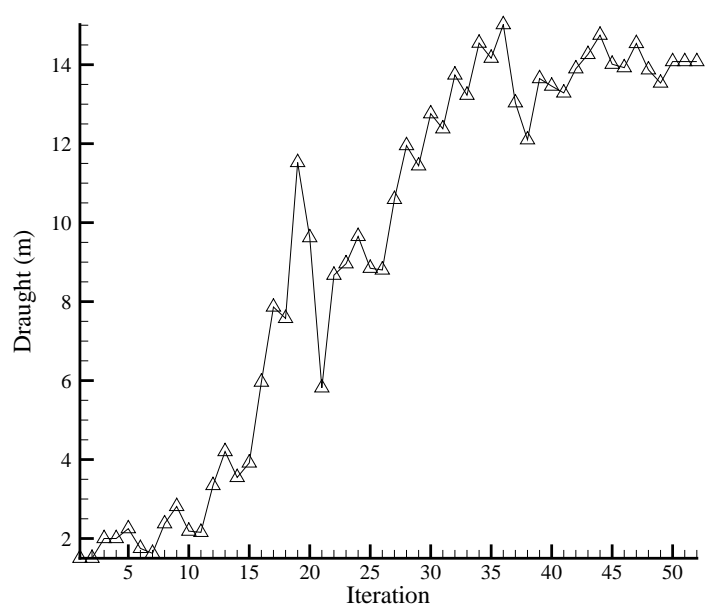

Fig. 7. Evolution of the draught with a passive PTO.

101-109.

Eidsmoen, H. (1998). Tight-moored amplitude-limited heaving buoy wave energy converter with phase control. Applied Ocean Research, 20, 157-167.

McCabe, A.P., Aggidis, G.A., and Widden, M.B. (2009). A pilot study into the optimization of the shape of a wave energy collector by genetic algorithm. Proceedings of the 8th European Wave and Tidal Energy Conference).

Nelder, J.A. and Mead, R. (1965). A simplex method for function minimization. Computer Journal, 7, 308-313.

Rawson, K. and Tupper, F. (1992). Basic ship theory, volume 1. Longman.

Ricci, P., Alves, M., Falco, A., and Sarmento, A. (2006). Optimisation of the geometry of wave energy converters. OTTI, International Conference Ocean Energy, Bremerhaven, Germany. 


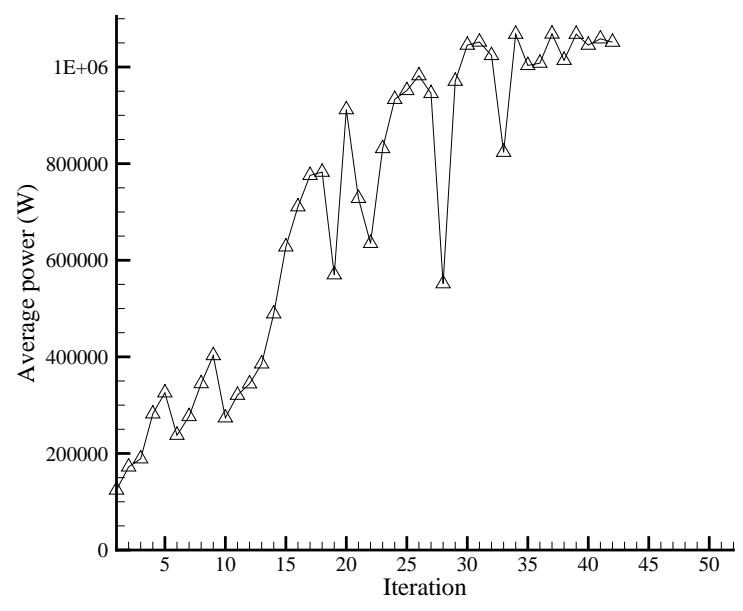

Fig. 8. Evolution of the average power with latching.

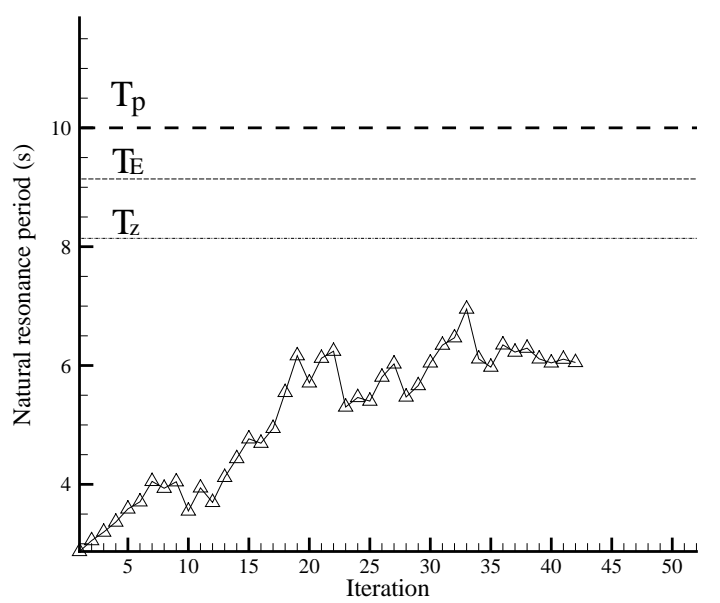

Fig. 9. Evolution of the resonance period with latching.

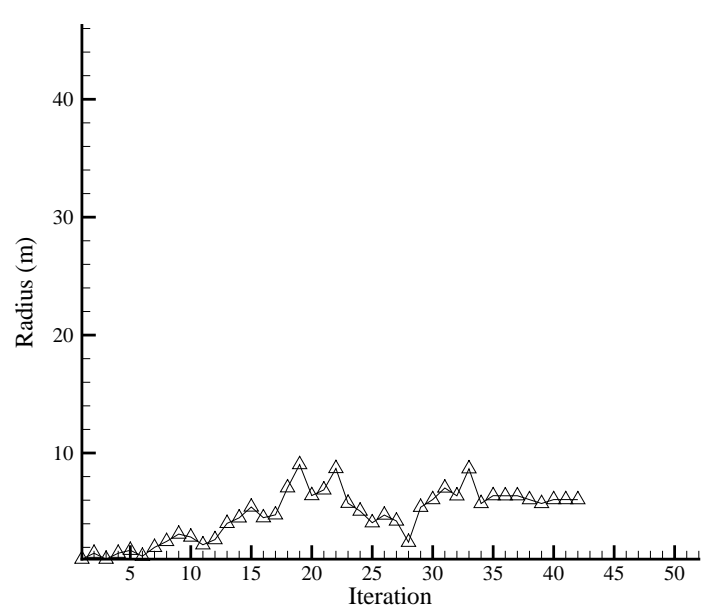

Fig. 10. Evolution of the radius with latching.

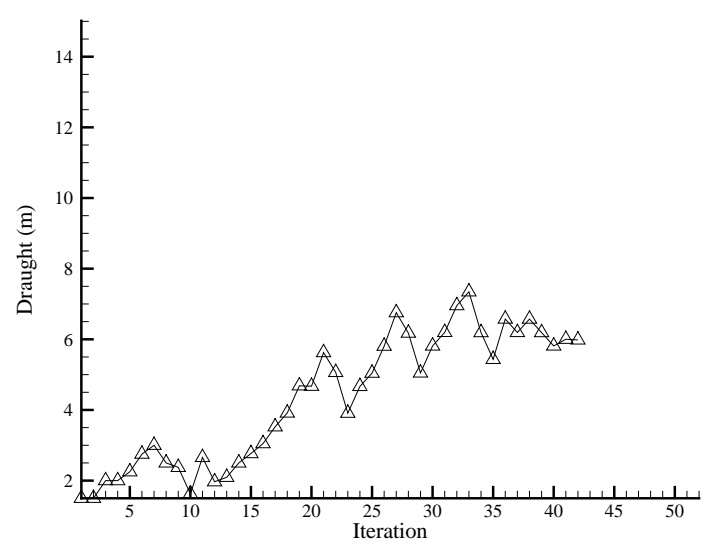

Fig. 11. Evolution of the draught with latching. 